\author{
Ivan O. Haefliger \\ Qian Chen \\ Douglas R. Anderson
}

\section{Effect of oxygen on relaxation of retinal pericytes by sodium nitroprusside}

Received: 27 September 1996 Revised version received: 21 January 1997 Accepted: 4 February 1997
I.O. Haefliger (

Laboratory for Ocular Pharmacology

and Physiology,

University Eye Clinic Basel,

Mittlere Strasse 91, PO Box,

CH-4012 Basel, Switzerland

Tel. +41-61-321-7777;

fax $+41-61-322-4001$

Q. Chen - D.R. Anderson

Department of Ophthalmology,

Bascom Palmer Eye Institute,

University of Miami School of Medicine,

Miami, FL 33101-6880, USA
Abstract - Background: This

study addresses whether oxygen modulates the relaxation induced in retinal pericytes by sodium nitroprusside (SNP), a nitric oxide (NO) donor that stimulates the $\mathrm{NO} /$ guanylate cyclase pathway. - Methods:Bovine retinal pericytes were cultured on silicone. On the silicone surface, basal pericyte contractile tone induces wrinkles. Drug-induced changes in pericyte contractile tone were assessed by changes in the number of wrinkles. The effects of $100 \%$ nitrogen (hypoxia) and $100 \%$ oxygen (hyperoxia) were studied on: (a) the basal tone of quiescent pericytes, (b) the relaxation to 3 and $10 \mu \mathrm{M}$ SNP or $1 \mu \mathrm{M}$ forskolin, and (c) the recontraction that followed the washout of $3 \mu \mathrm{M}$ SNP or $1 \mu \mathrm{M}$ forskolin. - Results: Neither hypoxia nor hy- peroxia had any apparent influence on pericyte basal tone, on forskolininduced relaxation, or on pericyte recontraction after a forskolininduced relaxation. In hypoxia, relaxations to SNP $3 \mu \mathrm{M}(P<0.05)$ and $10 \mu \mathrm{M}(P<0.01)$ were significantly more pronounced than in hyperoxia. Hypoxia also reduced the recontraction after an SNPinduced relaxation $(P<0.001)$. - Conclusion: Oxygen modulates the relaxation of bovine retinal pericytes evoked by SNP (guanylate cyclase-mediated), but not the relaxation induced by forskolin (adenylate cyclase-mediated). These results suggest that in the retinal capillary circulation an interaction between oxygen and the $\mathrm{NO} /$ guanylate cyclase pathway modulates pericyte tone, and thus potentially blood flow.

\section{Introduction}

Capillaries of the retina and optic nerve head are interconnected in a dense and continuous network $[2,3,5]$. They are made of an endothelium layer surrounded by pericytes of a mesenchymal origin $[10,11]$.

Pericytes are contractile cells which are relaxed by nitric oxide (NO) $[15,17]$. They can modify the diameter of a retinal capillary [24] and thus potentially flow within such a vessel $[18,22]$.

Blood flow in the retina and the optic nerve head is increased and decreased in response to hypoxia and hyperoxia, respectively [12]. NO, which is rapidly degraded by oxygen [25], maintains the ophthalmic circulation in a constant state of mid-vasodilation $[8,13,14]$. Oxygen, by modulating the relaxing effect of NO, could help control retinal capillary blood flow in keeping with local metabolic needs, as reflected in local partial pressures of oxygen [6].

Therefore, in this study, we investigated the effect of $100 \%$ nitrogen (hypoxia) and $100 \%$ oxygen (hyperoxia) on the relaxation/recontraction of bovine retinal microcirculation pericytes in culture. We showed an effect of oxygen tension when the NO donor, sodium nitroprusside (SNP), was present. 


\section{Materials and methods}

\section{Pericyte isolation and identification}

Retinal bovine pericytes were grown in culture and identified according to the method described by D'Amore $[6,7,15]$. In agreement with the principles of laboratory animal care, as well as specific national laws for the use of animals in experimentation, bovine eyes were obtained from Aries Scientific (Richardson, Tex.) and shipped to our laboratory on ice by an overnight express service. Thirty to forty retinas were dissected free, minced, and incubated for $1 \mathrm{~h}$ in phosphate-buffered saline containing $0.2 \%$ collagenase and $0.2 \%$ bovine serum albumin. After filtration through a Nitex mesh, cells were retrieved and rinsed by serial centrifugation $(800 \mathrm{~g})$, resuspended, and cultured in $75-\mathrm{cm}$ square flasks in Dulbecco's modified Eagle medium (DMEM) containing $10 \%$ fetal bovine serum, $50 \mathrm{mg} / \mathrm{ml}$ fungizone, and $1.25 \mu \mathrm{g} / \mathrm{ml}$ gentamicin. The cells cultured had the typical morphological appearance of pericytes [7] and stained positively for the antiganglioside antibody $3 \mathrm{G} 5$, which marks pericytes but not vascular smooth muscle cells [20]. These cultured pericytes did not express the glial fibrillar acidic protein found in cultured astrocytes [1]. In contrast to retinal pigment epithelium cells, they lacked the ability to phagocytize rod outer segments [19]. In contrast to endothelial cells, the main potential contaminant of retinal pericyte culture, they did not take up fluorescein-tagged low-density lipoprotein (LDL) [23]. After these general features of the pericytes cultured had been demonstrated [6], each lot of cells was checked by documenting binding of the antiganglioside $3 \mathrm{G} 5$ and the absence of cells that take up LDL.

\section{Culture of pericytes on silicone membrane}

First and second passages of bovine retinal pericytes were grown in DMEM for 3-7 days on a thin membrane of silicone, coating the bottom of a petri dish. When growing on the surface of the silicone these contractile cells induced small wrinkles that were visualized with a phase-contrast, inverted microscope (Zeiss, Oberkochen, Germany) connected to a video camera system (Panasonic, Osaka, Japan) and a printer (Mitsubishi, Cypress, Calif.) $[15,16]$. In these cultures, pericytes spontaneously exhibited contractile tone that produced a wrinkled silicone surface beneath them.

\section{Observation of pericytes}

To study the contractile function of the pericytes, the whole petri dish was placed into a closed transparent chamber that maintained the cells at a constant $37^{\circ} \mathrm{C}$ while allowing multiple fluid exchanges by suction-perfusion. In certain experiments the atmosphere in the chamber (ambient air) was saturated with oxygen or nitrogen. Once the dish was in the chamber, the DMEM solution was immediately replaced with a HEPES (pH 7.4)-buffered solution $(\mathrm{NaCl} 140 \mathrm{mM}$; $\mathrm{KCl} 5 \mathrm{mM}$; $\mathrm{CaCl} 21 \mathrm{mM}$; $\mathrm{MgCl} 21.5 \mathrm{mM}$; HEPES $5 \mathrm{mM}$; HEPES sodium salt $5 \mathrm{mM}$; glucose $10 \mathrm{mM}$ ), a solution that was then used in all subsequent experiments.

After $20 \mathrm{~min}$ of adaptation in this solution, pericytes were relaxed with either SNP (3 and $10 \mu \mathrm{M}$ ) or forskolin $(1 \mu \mathrm{M})$, two substances that stimulate the guanylate cyclase [15] and the adenylate cyclase pathway, respectively [9]. SNP and forskolin were used at concentrations that were about or slightly higher than the half maximal relaxing concentration $[9,15]$. The effect of oxygen concentration on the relaxation was studied.

In separate experiments, the spontaneous recontraction of pericytes after exposure and relaxation to $3 \mu \mathrm{M}$ SNP or $1 \mu \mathrm{M}$ forskolin (in normoxia) was studied. In these experiments, pericytes were first relaxed with $3 \mu \mathrm{M}$ SNP or $10 \mu \mathrm{M}$ forskolin. After $10 \mathrm{~min}$, when the maximal relaxing effect of drugs was reached, SNP and forskolin solutions were washed out and replaced by plain HEPESbuffered solution. The recovery of pericyte contractile tone was then observed, and the effect of oxygen concentration on the recovery was tested.

\section{Changes in oxygen concentrations}

Hypoxic and hyperoxic conditions were obtained by bubbling the HEPES-buffered solution for $10 \mathrm{~min}$ with $100 \%$ nitrogen and $100 \%$ oxygen, respectively, prior to its infusion in the petri dish. Once in the petri dish, the atmosphere surrounding the solution was immediately saturated with either $100 \%$ nitrogen or $100 \%$ oxygen. In preliminary experiments it was shown that, after bubbling for 10 min the petri dish solution with $100 \%$ nitrogen or $100 \%$ oxygen, the partial pressure of oxygen in the solution was $2 \%$ and $97 \%$, respectively, conditions which are clearly hypoxic and hyperoxic. Our experiments, specifically studied the effect of hypoxic, normoxic and hyperoxic conditions on the basal resting tone of pericytes, on the relaxation induced by either SNP or forskolin, and on the recontraction of pericytes after the wash-out of either SNP or forskolin.

\section{Assessment of the data}

Changes in pericyte contractile tone were quantified by counting the changes in the number of the wrinkles [15]. Measurements were usually taken at 2,5 , and $10 \mathrm{~min}$. In all experimental protocols, maximal effect was reached after $10 \mathrm{~min}$; therefore, only measurements taken at $10 \mathrm{~min}$ were compared. Results are expressed either as percentage loss of the number of wrinkles of pericyte's basal tone or as percentage recovery of the number of wrinkles after a relaxation induced by SNP $(3 \mu \mathrm{M})$ or forskolin $(1 \mu \mathrm{M})$. The data, which were normally distributed, are given as the mean and the standard error of the mean (mean \pm SEM), and the results are compared using an unpaired Student's $t$-test, with $P<0.05$ considered significant.

\section{Drugs}

Sodium nitroprusside, forskolin, fungizone, collagenase, HEPES, dimethylpolysiloxane, and bovine serum albumin were obtained from Sigma Chemical (St Louis, Mo.). Gentamicin and the fetal bovine serum were purchased at Gibco (Gaithersburg, Md.), and the oxygen and the nitrogen at Liquid Carbonics (Miami, Fla.). SNP was diluted in distilled water and made daily. Forskolin was diluted in alcohol and stored in stock solution $(-20 \mathrm{C})$.

\section{Results}

\section{Effect of oxygen on pericyte basal tone}

Exposing quiescent pericytes for $10 \mathrm{~min}$ to hypoxic or hyperoxic conditions had virtually no effect on pericyte basal contractile tone. Indeed, hypoxia or hyperoxia only evoked a slight and no significant decrease in the number of wrinkles of $1 \pm 6 \%(n=5)$, and $5 \pm 3 \%(n=4)$, respectively (Fig. 1). 


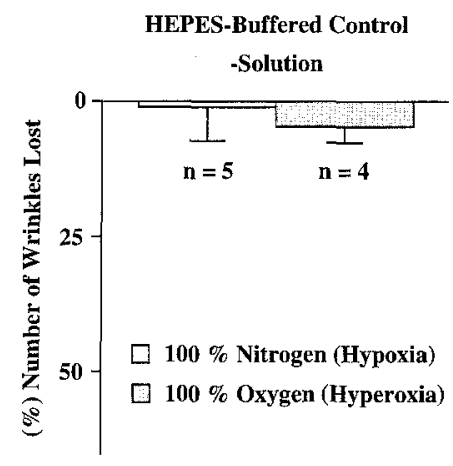

Fig. 1 Effect of 100\% nitrogen (hypoxia) vs 100\% oxygen (hyperoxia) on the basal tone of quiescent pericytes in a HEPES-buffered control solution. Neither hypoxia nor hyperoxia had an influence on pericyte basal tone

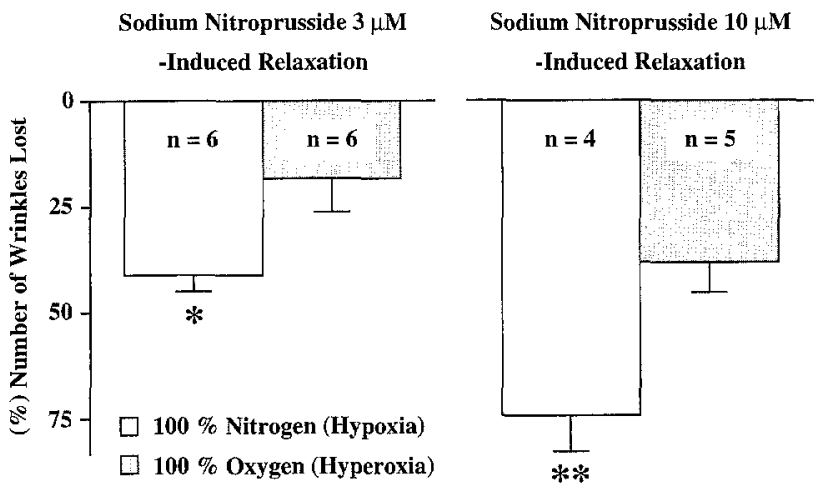

Fig. 2 Effect of $100 \%$ nitrogen (hypoxia) vs $100 \%$ oxygen (hyperoxia) on the relaxation of bovine retinal microcirculation pericytes to SNP $3 \mu \mathrm{M}$ and $10 \mu \mathrm{M}$. Relaxations were significantly greater in hypoxia than in hyperoxia. Unpaired Student's $t$-test, hypoxia vs hyperoxia: " $P<0.05$, "* $P<0.01$

Effect of hypoxia vs hyperoxia on pericyte relaxations to SNP or forskolin

In comparison to hyperoxic conditions, in hypoxia relaxations to both SNP $3 \mu \mathrm{M}(P<0.05)$ and SNP $10 \mu \mathrm{M}$ $(P<0.01)$ were significantly more pronounced (Fig. 2$)$. In hypoxia, SNP $3 \mu \mathrm{M}$ evoked $41 \pm 4 \%(n=6)$, and SNP $10 \mu \mathrm{M}$ evoked $74 \pm 8 \%(n=4)$ relaxations, while in hyperoxia relaxations to SNP $3 \mu \mathrm{M}$ and $10 \mu \mathrm{M}$ were only $19 \pm 8 \%(n=6)$ and $38 \pm 7 \%(n=5)$, respectively. In contrast, no significant difference could be detected between relaxations induced by forskolin $1 \mu \mathrm{M}$ in hypoxic and hyperoxic conditions (Fig. 3); in hypoxia forskolin $10 \mu \mathrm{M}$ evoked $44 \pm 7 \%(n=9)$ and in hyperoxia, $43 \pm 9 \%(n=4)$, relaxations. Likewise, the oxygen level had no effect on the basal contractile tone (Fig. 1).

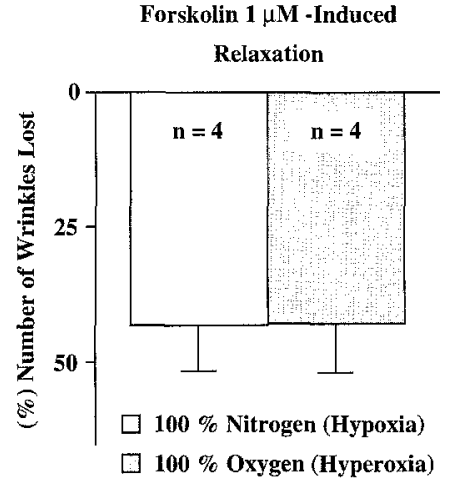

Fig. 3 Effect of $100 \%$ nitrogen (hypoxia) vs $100 \%$ oxygen (hyperoxia) on the relaxation of bovine retinal microcirculation pericytes to forskolin $1 \mu \mathrm{M}$. No difference was seen between hypoxia and hyperoxia

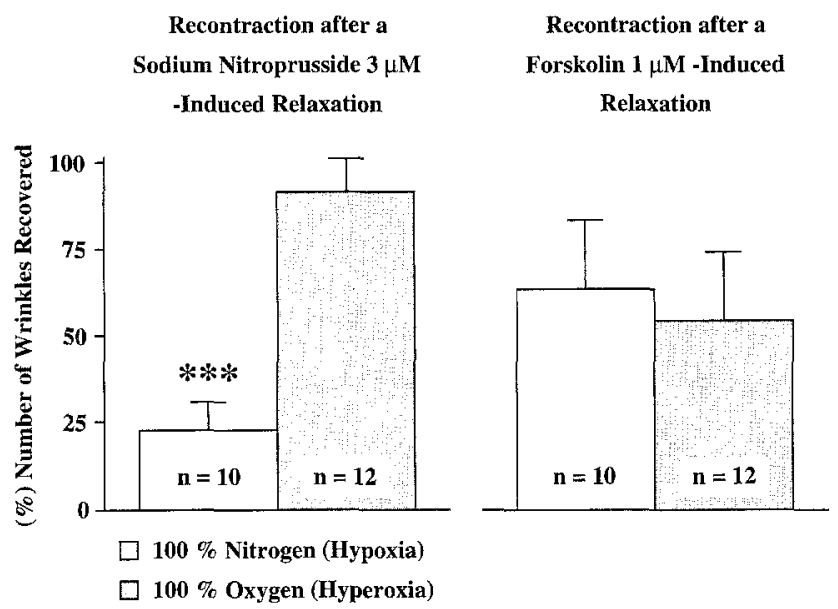

Fig. 4 Effect of 100\% nitrogen (hypoxia) vs $100 \%$ oxygen (hyperoxia) on recontraction of bovine retinal microcirculation pericytes following a relaxation induced either by SNP $3 \mu \mathrm{M}$ or forskolin $1 \mu \mathrm{M}$. The level of recontraction was assessed by the amount of wrinkles recovered by pericytes in a HEPES-buffered solution $10 \mathrm{~min}$ after the wash-out of SNP or forskolin. In contrast to forskolin, after a SNP-induced relaxation, recontraction was significantly lower in hypoxia than in hyperoxia. Unpaired Student's $t$-test, hypoxia vs hyperoxia: ${ }^{* * *} P<0.001$

Effect of hypoxia vs hyperoxia on pericyte recontractions after SNP or forskolin wash-out

After a $3 \mu \mathrm{M}$ SNP-induced relaxation and the wash-out of this drug, the spontaneous pericyte recontraction was strongly modulated by different oxygen concentrations (Fig. 4). In hypoxia $(23 \pm 8 \%, n=10)$ the recontraction after an SNP-induced relaxation was significantly lower $(P<0.001)$ than in hyperoxia $(91 \pm 10 \%, n=12)$. Such a difference could not be observed in pericyte recontraction which followed a $1 \mu \mathrm{M}$ forskolin-induced relaxation (hypoxia $64 \pm 20 \%, n=6$; hyperoxia $54 \pm 20 \%, n=6$; Fig. 4). 


\section{Discussion}

The present study on bovine retinal microcirculation pericytes demonstrates that after exposure to the NO donor SNP, both the relaxation and the recontraction that follows wash-out are strongly modulated by the oxygen concentrations. Indeed, in hypoxia relaxations to SNP were significantly more pronounced, and recontraction significantly less marked, than in hyperoxia (Figs. 2, 4). In contrast, forskolin-evoked relaxation and spontaneous recontraction after forskolin exposure were similar in hypoxia or in hyperoxia (Figs. 3, 4).

The oxygen modulation of SNP pericyte relaxation or recontraction does not appear to result from an unspecific direct relaxing or contracting effect of oxygen, as neither basal nor forskolin-modulated contractile tone of pericytes was affected by hypoxia or hyperoxia (Figs. 1, 3, 4). These results show that the oxygen effect on the relaxation or recovery of pericyte contractile tone (Figs. 2,4) is directly linked to the pharmacological properties of SNP.

In pericytes, SNP is known to evoke a relaxation through the stimulation of the guanylate cyclase pathway [15], while forskolin relaxes pericytes by the activation of the adenylate cyclase pathway [9]. Therefore, these results demonstrate that it is most likely by interacting with the NO/guanylate cyclase pathway that oxygen modulates the SNP-induced effect on pericytes', contractile tone. As NO is known to be rapidly autoxidized, it is likely that by modulating the half-life of NO, oxygen could indirectly modulate the relaxation of pericytes induced by NO [21, 25]. However, further investigations need to be conducted in order to clarify the exact mechanism by which oxygen modulates the effect of SNP on retinal pericytes.

In conclusion, these results clearly show that the contractile tone of bovine retinal pericytes in response to the NO donor, SNP, is strongly modulated by different concentrations of oxygen. Because the retinal vascular bed is constantly under the influence of NO, an interaction between oxygen and the $\mathrm{NO} /$ guanylate cyclase pathway by modulating pericyte tone - could potentially influence flow within the retinal capillary network $[4,12]$.

Acknowledgements This work was supported in part by US Public Health Service Research Grant RO1 EY 10465, awarded by the National Eye Institute, Bethesda, Maryland, USA. Dr. Douglas R. Anderson has been given a Senior Scientific Investigator's Award by Research to Prevent Blindness, Inc., New York, NY, USA.

Dr. Ivan O. Haefliger was supported by grants of the Sociéte Suisse de Bourses en Médecine et Biologie, the Fondation Florian Verrey, the Fondation Eugenio Litta, and the Swiss National Science Research Foundation (32-42564.94). The authors thank E. Barry Davis for his unique support during the course of this study.

\section{References}

1. Aotaki-Keen AE, Harvey AK, de Juan E, Hjelmand LM (1991) Primary culture of human retinal glia. Invest Ophthalmol Vis Sci 32: 1733-1738

2. Anderson DR (1970) Vascular supply to the optic nerve of primates. Am $\mathbf{J}$ Ophthalmol 60: 341-351

3. Anderson DR (1970) Ultrastructure of the optic nerve head. Arch Ophthalmol 83: 63-73

4. Anderson DR (1996) Glaucoma, cap illaries, and pericytes. 1. Blood flow regulation. Ophthalmologica 210 : 257-262

5. Anderson DR, Braverman S (1976) Optic disc vasculature. Am J Ophthalmol 82: 165-174

6. Anderson DR, Davis EB (1996) Glaucoma, capillaries, and pericytes. 2 . Identification and characterization of retinal pericytes in culture. Ophthalmologica 210: 263-268

7. D'A more PA (1990) Culture and study of pericytes. In Piepr HM (ed) Cell culture techniques in cardiovascular research. Springer, Berlin Heidelberg New York, pp 299-314
8. Donati G, Pournaras CJ, Munoz JL, Poitry S, Poitry-Yamate CL, Tsacopoulos M (1995) Nitric oxide controls arteriolar tone in the retina of the miniature pig. Invest Ophthalmol Vis Sci 36: 2228-2237

9. Ferrari-Dileo G, Davis EB, Anderson DR (1992) Effects of cholinergic and adrenergic agonists on adenylate cyclase activity of retinal microvascular pericytes in culture. Invest Ophthalmol Vis Sci 33: 42-47

10. Frank RN, Dutta S, Mancini MA (1987) Pericyte coverage is greater in the retinal than in the cerebral capillaries of the rat. Invest Ophthalmol Vis Sci 28: 1086-1091

11. Frank RN, Turczyn TJ, Das A (1990) Pericyte coverage of retinal and cerebral capillaries. Invest Ophthalmol Vis Sci. 31: 999-1007

12. Haefliger IO, Anderson DR (1996) Blood-flow regulation in the optic nerve head. In: Ritch R, Shields MB, Krupin T (eds) The Glaucomas, $2^{\text {nd }}$ edition. Mosby-Year Book, Inc., St Louis, MI, pp 189-197

13. Haefliger IO, Flammer J, Lüscher TF (1992) Nitric oxide and endothelin-1 are important regulators of the human ophthalmic artery. Invest Ophthalmol $V$ is Sci 33: $2340-2343$
14. Haefliger IO, Meyer P, Flammer J, Lüscher TF (1994) The vascular endothelium as a regulator of the ocular circulation: a new concept in ophthalmology? Surv Ophthalmol 39: 123132

15. Haefliger IO, Zschauer A, Anderson DR (1994) Relaxation of retinal pericytes contractile tone through the nitric oxide cyclic guanosine monophosphate pathway. Invest Ophthalmol Vis Sci 35: 991-997

16. Harris AK, Wild P, Stopak D (1980) Silicone rubber substrata: A new wrinkle in the study of cell locomotion. Science 208: 177-179

17. Kelley C, D'Amore P, Hechtman HB, Shepro D (1987) Microvascular pericyte contractility in vitro: Comparison with other cells of the vascular wall. J Cell Biol 104: 483-490

18. Lipowsky HH, Kolvalcheck S, Zweifach BW (1978) The distribution of blood rheological parameters in the microvasculature of cat mesentery. Circ Res 43: 738-749 
19. McLaren MJ, Inana G, Li CY (1993) Double fluorescent vital assay of phagocytosis by cultured retinal pigment epithelial cells. Invest Ophthal$\mathrm{mol}$ Vis Sci 34: 317-326

20. Nayak RC, Berman AB, George KL, Eisenbarth GS, King GL (1988) A monoclonal antibody (3G5)-defined ganglioside antigen is expressed on the cell surface of microvascular pericytes. J Exp Med 167: 1003-1015
21. Rubanyi GM, Vanhoutte PM (1986) Superoxide anions and hyperoxia inactivate endothelium-derived relaxing factor. Am J Physiol 250: H822$\mathrm{H} 827$

22. Tilton RG, Kilo C, Williamson JR, Murch DW (1979) Differences in pericyte contractile function in rat cardiac and skeletal muscle microvasculatures. Microvase Res 18: 336352

23. Voyata J, Via D, Butterfield C, Zetter B (1984) Identification and isolation of endothelial cells based on their increased uptake of acetylated-low density lipoproteins. J Cell Biol 99: 2034-2040
24. Wallow IH, Bindley CD, Reboussin DM, Gange SJ, Fisher MR (1993) Systemic hypertension produces pericyte changes in retinal capillaries. Invest Ophthalmol Vis Sci 34: 420-430

25. Wink DA, Beckman JS, Ford PC (1996) Kinetics of nitric oxide reaction in liquid and gas phase. In: Freelish $\mathrm{M}$, Stamler S (eds) Methods in Nitric Oxide Research. John Wiley \& Sons, Chichester, England, pp 29-37 